\title{
082 AN INTERVENTION STRATEGY FOR UNDERWEIGHT FILIPINO YOUNG CHILDREN: COMPLEMENTARY FEEDING AND NUTRITION EDUCATION
}

Julieta Bueta Dorado. Nutritional Assessment and Monitoring Division, Food and Nutrition Research Institute-DOST, Taguig City, Philippines

\subsection{6/bmjopen-2015-forum2015abstracts.82}

Background Malnutrition rates increase between six and 18 months, the period of complementary feeding. The 2011 Updating Survey of the Food and Nutrition Research Institute-DOST revealed a non-significant decrease in the proportion of underweight Filipino children aged $0-5$ years (20.6 percent to 20.2 percent) from 2008 to 2011 (FNRI-DOST, 2011).

Objectives This study aimed to reduce the underweight prevalence among 6-35 months old children and increase nutrition knowledge of mothers/caregivers after the intervention.

Methods A quasi-experimental design was used in the evaluation study with a total of 816 underweight children, 719 in the intervention and 97 in the non-intervention group. The intervention consisted of 120-day complementary feeding among children using the local-based complementary foods, and nutrition education among mothers/caregivers. The non-intervention group were fed with similar complementary foods after the intervention. Baseline and endline weights and heights of the children were taken using calibrated beam balance (Detecto) weighing scale and microtoise/infantometer, respectively. Profile of children and households, knowledge of mothers/caregivers, intakes of vitamins and incidence of illness among children were also collected. Parametric and non-parametric tests were employed in data analysis.

Result The prevalence of underweight children decreased significantly in the intervention (from 96.7 percent to 82.1 percent) compared to the non-intervention group (from 100 percent to 94.9 percent). Mean scores on nutrition knowledge among mothers/caregivers increased significantly at endline in the intervention group.

Conclusion The feeding intervention contributed to the improvement of the nutritional status of 6-35 months old children. Likewise, there was an improvement in the nutrition knowledge of mothers/caregivers. The intervention strategy provided policy directions for local government units to adopt the intervention for children 6-35 months old as a component of the local health and nutrition services. 